\title{
The Development and Interaction of Terrorist and Fanatic Groups
}

\author{
Erika T. Camacho, \\ School of Mathematical \& Natural Sciences, Arizona State University, \\ 4701 W. Thunderbird Rd, Glendale, AZ, 85306, USA, \\ and \\ Mathematical, Computational and Modeling Sciences Center, \\ Arizona State University, P.O. Box 871904, Tempe, AZ, 85287-1904, USA \\ tel: 602-543-8156, fax: 602-543-6073, erika.camacho@asu.edu
}

April 2, 2013

\begin{abstract}
Through the mathematical study of two models we quantify some of the theories of codevelopment and co-existence of focused groups in the social sciences. This work attempts to develop the mathematical framework behind the social sciences of community formation. By using well developed theories and concepts from ecology and epidemiology we hope to extend the theoretical framework of organizing and self-organizing social groups and communities, including terrorist groups. The main goal of our work is to gain insight into the role of recruitment and retention in the formation and survival of social organizations. Understanding the underlining mechanisms of the spread of ideologies under competition is a fundamental component of this work. Here contacts between core and non-core individuals extend beyond its physical meaning to include indirect interaction and spread of ideas through phone conversations, emails, media sources, and other similar mean. This work focuses on the dynamics of formation of interest groups, either ideological, economical or ecological and thus we explore the questions such as, how do interest groups initiate and co-develop by interacting within a common environment and how do they sustain themselves? Our results show that building and maintaining the core group is essential for the existence and survival of an extreme ideology. Our research also indicates that in the absence of competitive ability (i.e., ability to take from the other core group or share prospective members) the social organization or group that is more committed to its group ideology and manages to strike the right balance between investment in recruitment and retention will prevail. Thus under no cross interaction between two social groups a single tradeoff (of these efforts) can support only a single organization. The more efforts that an organization implements to recruit and retain its members the more effective it will be in transmitting the ideology to other vulnerable individuals and thus converting them to believers.
\end{abstract}

keywords: competing interest groups; extreme ideology; epidemiological model

\section{Introduction}

The events in recent years have triggered a desire to understand fanatic behavior on a global scale. Behind the formation of most rebellious forces there is a period of competition for support with other similar forces, where groups try to build themselves from the bottom up with the main objective of increasing their strength (which depends on many variables). Under the assumption of constant environmental conditions, equal resources and group capacities among other relevant factors the only thing that could cause differences in strength would be the critical mass of each respective group. In this very simplistic setting, most anti-fanatic policy makers would be in agreement with "the common sense view that the most effective counterterrorist approach consists of depriving fanatic groups from recruitment sources" while discouraging some of there current members [8]. 
In such a setting where recruitment and retention are vital to the group existence, it is crucial to understand and mathematically formulate the mechanism by which fanatic groups establish a critical mass. An epidemiological model with homogeneous mixing can help us accomplish this.

There are numerous examples where individuals have influenced one another even though they have never met in person. This can happen for example via a phone conversation or electronic mail. It is this type of contact that we assume in our work and thereby attempt to develop some qualitative framework that might shed light on possible ways of fighting extreme ideologies (fanaticism) that might hinder mankind. In an effort to better understand the spread of ideas in a vulnerable population we have extended the work of Castillo-Chavez and Song to include interaction between two interest groups [8]. We assume that interaction includes competition, cooperation, and synergy. Interest groups may include but are not limited to those which are socially active, focused politically, unions, educational groups, informal social groups, professional societies, fanatic groups, terrorists, and religious groups. In particular, we study the spread of ideas as an epidemiological contact process where the populations in each subclass are assumed to be different but homogeneous within each subclass. Transmission of ideas is not an individualto-individual process but rather part of a group effort A key element in this work is the trade off between recruitment of individuals into the core population and retention. Here contacts between core and non-core individuals extend beyond its physical meaning to include indirect interaction and spread of ideas through phone conversations, emails, media sources, and other similar means.

Our mathematical work is not intended to predict the evolution of any two specific fanatic ideologies in our current society but rather to give a very general qualitative measure of the forces behind fanatic acts and the social structure that facilitates the existence of such groups [2],[18],[19],[20],[21], $[23],[24],[26]$. It is important to note that the concepts of ideology, fanatic, and terrorist behavior are too vague and complex to be defined in this work. In this work we hope to gain some insight into possible mechanisms by which social organizations form due to a shared common ideology among its potential members or due to group pressure. Understanding this will help us identify the conditions and limiting factors that allow certain social groups to prevail.

Since we are considering a homogeneous population, our study does not explicitly incorporate heterogeneity; however, group appeal (or individual preference) which is key under competition of social groups, especially fanatic groups, is incorporated via recruitment. The generalization of recruitment in this setting is growth due to attraction (or maybe even coercion). Extending the work of Castillo-Chavez and Song, we develop a mathematical framework to systematically study the transmission of ideas under competition from a population dynamics approach. We build-on their model and consider multiple ideologies. We imagine that fanatics can only draw support to their extreme views and convert individuals from susceptible subpopulations, the core groups. Only the individuals in the core subpopulations are vulnerable and receptive to transmission. The recruitment process is modeled based on the work of [8],[11],[13],[25].

We propose two models describing the dynamics in the evolution of two organizations. One with interaction between the core groups and one with no interaction (i.e., with and without competitive ability/ cross-recruitment between the competing ideologies), which we will refer to as the cross interaction model and non-cross interaction model, respectively. Following [8] and drawing from [20]'s triadic system we imagine that the total population, $N(t)$, is divided into the non-core population, defined as $G(t)$, and the core population(s), defined as $C_{1}(t), C_{2}(t)$, and $C(t)$. The core populations are made up of vulnerable (or naive) individuals, $S(t)$ or $S_{i}(t)$, semi-fanatics, $E_{i}(t)$, and fanatics, $F_{i}(t)$ for $i=1,2$, in other words individuals who have been introduced, have adopted, and are fully committed to ideology $i$, respectively. In the cross interaction model there is only one core subpopulation, $C(t)=S(t)+E_{1}(t)+E_{2}(t)+F_{1}(t)+F_{2}(t)$, as ideology 1 and 2 share some characteristics in the initial stages of evolution of their respective organization but diverge as 
their followers become more committed. The parallel characteristics allows for these organizations to cross-recruit and share prospective members. The non-cross interaction model, has two core groups, $C_{i}(t)=S_{i}(t)+E_{i}(t)+F_{i}(t), i=1,2$, consisting of ideology 1 and 2, respectively. In both models, the group $G(t)$ serves as the recruitment pool for the core group(s).

The main goal of this work is to extend the theoretical framework of organizing and selforganizing social groups/communities by using well developed theories and concepts from ecology and epidemiology. In particular, to gain insight of the role of recruitment and retention in the formation and survival of social organizations, understanding the underlining mechanisms of the spread of ideologies under competition will be fundamental.

\section{Description of the non-cross interaction model}

In the non-cross interaction model, the movement of individuals into various fanatic classes within each core group is modeled identical to that of [8], except that now there are two core groups, $C_{1}(t)=S_{1}(t)+E_{1}(t)+F_{1}(t)$ and $C_{2}(t)=S_{2}(t)+E_{2}(t)+F_{2}(t)$. The $i$ th core subpopulation, $C_{i}(t)$, contains three classes: the vulnerable subpopulation, $S_{i}(t)$, composed of individuals who have not yet been converted but are part of the core group; the semi-fanatic group, $E_{i}(t)$, which includes all the members that have just been converted and are not fully committed; and, the fanatic subpopulation, $F_{i}(t)$, composed of individual which are fully committed to the extreme ideology. The core groups $C_{1}(t)$ and $C_{2}(t)$ recruit some portions $q$ and $(1-q)$ of individuals from the general population, $G(t)$, respectively. From there individuals move to the semi-fanatic and the fanatic classes by interacting with members of these respective groups. Assuming homogeneous mixing between all groups, the governing equations defining this process are

$$
\begin{aligned}
\frac{d G}{d t} & =\Lambda-\frac{q \beta_{1} G C_{1}}{N}-\frac{(1-q) \beta_{1} G C_{2}}{N}+\gamma_{1} S_{1}+\gamma_{2} E_{1}+\gamma_{3} F_{1}+\alpha_{1} S_{2}+\alpha_{2} E_{2}+\alpha_{3} F_{2}-\mu G,(1) \\
\frac{d S_{1}}{d t} & =\frac{q \beta_{1} G C_{1}}{N}-\frac{\beta_{2} S_{1}\left(E_{1}+F_{1}\right)}{C_{1}}-\gamma_{1} S_{1}-\mu S_{1} \\
\frac{d S_{2}}{d t} & =\frac{(1-q) \beta_{1} G C_{2}}{N}-\frac{\rho_{2} S_{2}\left(E_{2}+F_{2}\right)}{C_{2}}-\alpha_{1} S_{2}-\mu S_{2} \\
\frac{d E_{1}}{d t} & =\frac{\beta_{2} S_{1}\left(E_{1}+F_{1}\right)}{C_{1}}-\frac{\beta_{3} E_{1} F_{1}}{C_{1}}-\mu E_{1}-\gamma_{2} E_{1} \\
\frac{d E_{2}}{d t} & =\frac{\rho_{2} S_{2}\left(E_{2}+F_{2}\right)}{C_{2}}-\frac{\rho_{3} E_{2} F_{2}}{C_{2}}-\mu E_{2}-\alpha_{2} E_{2} \\
\frac{d F_{1}}{d t} & =\frac{\beta_{3} E_{1} F_{1}}{C_{1}}-\gamma_{3} F_{1}-\mu F_{1} \\
\frac{d F_{2}}{d t} & =\frac{\rho_{3} E_{2} F_{2}}{C_{2}}-\alpha_{3} F_{2}-\mu F_{2}
\end{aligned}
$$

where all parameters are nonnegative and where the total population, $N(t)$, and the core groups $C_{1}(t)$ and $C_{2}(t)$, are given by

$$
\begin{aligned}
N & =G+S_{1}+S_{2}+E_{1}+E_{2}+F_{1}+F_{2} \\
C_{1} & =S_{1}+E_{1}+F_{1} \\
C_{2} & =S_{2}+E_{2}+F_{2} .
\end{aligned}
$$

Since our variables define groups of individuals in the various populations, initially they must satisfy

$$
G(0)>0, S_{i}(0) \geq 0, E_{i}(0) \geq 0, F_{i}(0) \geq 0 .
$$


A pictorial illustration of this model is given in Figure1.

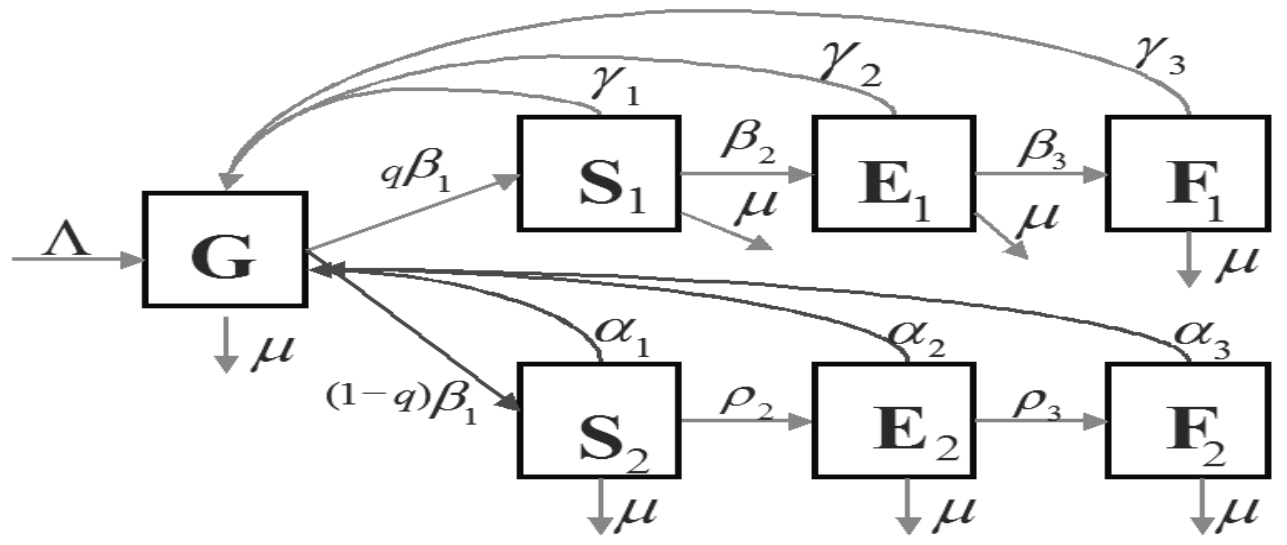

Figure 1: Flow diagram for the non-cross interaction model.

We assume a constant birth rate, $\Lambda$, into the general population. The core groups, $C_{i}(t)$, $i=1,2$, build their membership from the general population, $G(t)$, and convert them at the rates $q \beta_{1} G C_{1} / N$ and $(1-q) \beta_{1} G C_{1} / N$, respectively. The recruitment effect, measured by $\beta_{1}$ includes the attractiveness of the core group, the resonance with the ideologies of this group, and the likelihood to join based on exposure to these ideas. Thus the parameter $\beta_{1}$ serves as a measurement of the relative strength of the recruitment force of the core groups. Each $\beta_{1}$ together with the parameter $q$ defines the investment efforts that each fanatic group puts into recruiting their members. Core group, $C_{1}(t)$, invest $q \beta_{1}$ efforts into recruiting while core group $C_{2}(t)$ invests $(1-q) \beta_{1}$. Thus the relative sizes of $q$ and $(1-q)$ determines which group invests more in recruitment. The parameter $q$ illustrates how our model assumes competition between two focused groups where one focused group cannot increase its recruitment effort without the other decreasing its effort, except by increasing its numbers. Since our model follows an epidemiological approach, all conversions into a more active class occur only through effective contacts and they are assumed to be proportional to the number of contacts per unit time.

Once an idea has infiltrated the general population and a core group is formed (i.e., once an epidemic has started), individuals can return to the previous less active subgroup (and hence ultimately end up in the general class, $G(t))$ through a recovery process. The rate of return to the $G(t)$ class depends on the number of individuals in the respective $E_{i}(t)$ or $F_{i}(t)$ class. Ideology groups tend to lose numbers when there is lack of direction, which is captured by these groups. We imagine that the more fanatic an individual becomes the less likely he/she is to move to a less active group (because he/she has a stronger sense of commitment to his/her ideology). We assume that the residence time in the core groups are exponentially distributed and we let $\gamma_{i}$ and $\alpha_{i}$ denote the percapita recovery rates for each subgroup in the core subpopulations $C_{i}(t)$ for $i=1,2$, respectively. The average residence time for susceptibles, $S_{1}(t)$, semi-fanatics $E_{1}(t)$, and fanatics, $F_{1}(t)$, in the core group, $C_{1}(t)$ are $1 /\left(\gamma_{i}+\mu\right)$ for $i=1,2,3$, respectively. Similarly, the average residence time 
for the corresponding subpopulations in the core group, $C_{2}(t)$, are $1 /\left(\alpha_{i}+\mu\right)$ for $i=1,2,3$. The assumption that the more fanatic individuals have stronger ties translates to longer time spent in the more active group. Hence we assume $1 /\left(\alpha_{3}+\mu\right) \gg 1$ and $1 /\left(\gamma_{3}+\mu\right) \gg 1$. The investment efforts in retention (that is, of keeping members within a particular group), of each focused group is measured by the minimum recovery rate, $\gamma=\min \left\{\gamma_{1}, \gamma_{2}, \gamma_{3}\right\}$ and $\alpha=\min \left\{\alpha_{1}, \alpha_{2}, \alpha_{3}\right\}$.

The equation $\dot{N}(t)=\Lambda-\mu N$, governs the dynamics of the total population, $N(t)$, and thus in the long run the total population is given by $\Lambda / \mu$, i.e., $\lim _{t \rightarrow \infty} N(t)=\Lambda / \mu$. We use the relationship between the dynamics of non-autonomous systems and their natural limiting systems to reduce the dimension of our system. We replace $N(t)$ and $G(t)$ by $\Lambda / \mu$ and $\Lambda / \mu-\left(C_{1}(t)+C_{2}(t)\right)$, respectively, in the equations 2-7. Hence, we obtain the dynamically equivalent system,

$$
\begin{aligned}
\frac{d S_{1}}{d t} & =-\frac{S_{1} \beta_{2}\left(F_{1}+E_{1}\right)}{C_{1}}+q \beta_{1}\left(C_{1}-C_{1}^{2}-C_{1} C_{2}\right)-S_{1}\left(\gamma_{1}+\mu\right) \\
\frac{d S_{2}}{d t} & =-\frac{S_{2} \rho_{2}\left(F_{2}+E_{2}\right)}{C_{2}}+(1-q) \beta_{1}\left(C_{2}-C_{2}^{2}-C_{1} C_{2}\right)-S_{2}\left(\alpha_{1}+\mu\right) \\
\frac{d E_{1}}{d t} & =\frac{\beta_{2} S_{1}\left(E_{1}+F_{1}\right)}{C_{1}}-\frac{\beta_{3} E_{1} F_{1}}{C_{1}}-\mu E_{1}-\gamma_{2} E_{1} \\
\frac{d E_{2}}{d t} & =\frac{\rho_{2} S_{2}\left(E_{2}+F_{2}\right)}{C_{2}}-\frac{\rho_{3} E_{2} F_{2}}{C_{2}}-\mu E_{2}-\alpha_{2} E_{2}, \\
\frac{d F_{1}}{d t} & =\frac{\beta_{3} E_{1} F_{1}}{C_{1}}-\gamma_{3} F_{1}-\mu F_{1} \\
\frac{d F_{2}}{d t} & =\frac{\rho_{3} E_{2} F_{2}}{C_{2}}-\alpha_{3} F_{2}-\mu F_{2}
\end{aligned}
$$

where

$$
\begin{aligned}
& C_{1}=S_{1}+E_{1}+F_{1} \\
& C_{2}=S_{2}+E_{2}+F_{2} .
\end{aligned}
$$

\subsection{Core Population}

We analyze the long term sustainability of the core population by utilizing the idea of the basic reproductive number from epidemiology. With this underlining framework we can conclude that for certain parameter values, the model predicts the extinction of both core subpopulations, $C_{1}(t)$ and $C_{2}(t)$. Likewise we can show when the core population will persist.

Theorem 2.1 (i) Assume $\gamma=\min \left\{\gamma_{1}, \gamma_{2}, \gamma_{3}\right\}$ and $R_{1, \min } \equiv \frac{q \beta_{1}}{\gamma+\mu}<1$. Then $\lim _{t \rightarrow \infty} C_{1}(t) \rightarrow 0$.

(ii) Assume $\alpha=\min \left\{\alpha_{1}, \alpha_{2}, \alpha_{3}\right\}$ and $R_{2, \min } \equiv \frac{(1-q) \beta_{1}}{\alpha+\mu}<1$. Then $\lim _{t \rightarrow \infty} C_{2}(t) \rightarrow 0$.

(iii) Assume $\tau=\min \left\{\gamma_{1}, \gamma_{2}, \gamma_{3}, \alpha_{1}, \alpha_{2}, \alpha_{3}\right\}$ and $R_{\text {min }} \equiv \frac{\beta_{1}}{\tau+\mu}<1$ Then $\lim _{t \rightarrow \infty} C(t) \rightarrow 0$. 
Proof: Observing that $C_{i}=S_{i}+E_{i}+F_{i}$ for $i=1,2$, we calculate the change in the first core population $C_{1}$ as

$$
\begin{aligned}
\frac{d C_{1}}{d t} & =q \beta_{1} C_{1}\left(1-\left(C_{1}+C_{2}\right)\right)-\left(\gamma_{1}+\mu\right) S_{1}-\left(\gamma_{2}+\mu\right) E_{1}-\left(\gamma_{3}+\mu\right) F_{1} \\
& \leq q \beta_{1} C_{1}\left(1-\left(C_{1}+C_{2}\right)\right)-(\gamma+\mu) C_{1} \\
& \leq q \beta_{1} C_{1}-(\gamma+\mu) C_{1} \\
& =-(\gamma+\mu) C_{1}\left(1-\frac{q \beta_{1}}{\gamma+\mu}\right) \\
& <0
\end{aligned}
$$

where the last inequality holds because of the assumptions in (i). A similar proof shows that $d C_{2} / d t<0$ under (ii). Thus we see that the core populations will die out under the conditions of this theorem.

For part (iii), we observe that

$$
\begin{aligned}
\frac{d C_{1}}{d t}+\frac{d C_{2}}{d t} & \leq \beta_{1} q\left(C_{1}\left(C_{1}-1\right)-C_{2}\left(C_{2}-1\right)\right)-(\tau+\mu) C_{1}-(\tau+\mu) C_{2}+C_{2} \beta_{1}\left(1-C_{1}-C_{2}\right) \\
& =\beta_{1} q C_{1}\left(1-\left(C_{1}+C_{2}\right)\right)+(1-q) \beta_{1} C_{2}\left(1-\left(C_{1}+C_{2}\right)\right)-(\tau+\mu)\left(C_{1}+C_{2}\right) \\
& \leq \beta_{1} q C_{1}+(1-q) \beta_{1} C_{2}-(\tau+\mu)\left(C_{1}+C_{2}\right) \\
& =-C_{1}(\tau+\mu)\left(\frac{-\beta_{1} q}{\tau+\mu}+1\right)-C_{2}(\tau+\mu)\left(1-\frac{(1-q) \beta_{1}}{\tau+\mu}\right) \\
& =-C_{1}(\tau+\mu)\left(1-R_{\text {min }}\right)-C_{2}(\tau+\mu)\left(1-(1-q) R_{\text {min }}\right) .
\end{aligned}
$$

Because $0 \leq q \leq 1$, the conclusion of (iii) follows immediately.

The death of the core population also corresponds to the first of three equilibria where there is no established ideology for the non-cross recruiting model. These equilibria correspond to a social state where no fanatic organization is present. In the spirit of evoking mathematical epidemiology in this work we will refer to these three equilibria as disease-free equilibria $(D F E)$.

$$
\begin{array}{ll}
D F E_{0}: & \left(S_{1}, S_{2}\right)=(0,0) \\
D F E_{1}: & \left(S_{1}, S_{2}\right)=\left(1-\frac{\gamma_{1}+\mu}{q \beta_{1}}, 0\right) \\
D F E_{2}: & \left(S_{1}, S_{2}\right)=\left(0,1-\frac{\alpha_{1}+\mu}{\beta_{1}(1-q)}\right) .
\end{array}
$$

In $D F E_{1}$ and $D F E_{2}$, we observe that only one of the ideologies has a non-zero susceptible class from which it may draw to establish its organization. This indicates that only one fanatic group will persist under constant environmental conditions as the model does not account for external environmental changes that can perturb the system. Hence this model exhibits the competitive exclusion principle (CEP) which can be thought of as stating that two species (in this case social organizations) cannot coexist unless their limiting factors differ and are independent [15]. In the context of this work one could think of the core subpopulations, $C_{1}(t)$ and $C_{2}(t)$, as the species and the general population group, $G(t)$, as the limiting factor (the entity that each focused group feeds from to grow and increase its membership).

The work on the CEP goes back to Grinnell $(1904,1917)$, and it was first formalized by Volterra in 1926 when he showed that only one species can live of a single resource [6],[15]. The disagreement 
between the mathematical validity of this principle and the ecological observations of diverse species supported by a few resources has provided continued interest in the CEP. Since Volterra the theory of CEP has been extended to consider multiple species and resources with various trophic elements and the effect of variable environmental conditions on these types of predator-prey relations [28]. The fueled interest in the CEP lies primarily in the desire to understand the mechanisms that maintain the earth's vast biodiversity [16]. Our interest in this principle comes from the possibility of finding the crucial factors for coexistence and fitness advantage of organizations.

From the disease-free equilibria, we will be able to obtain the basic reproductive number, which quantifies how successful an ideology is in thriving. In epidemiological terms, the basic reproductive number gives the number of secondary infections caused by a primary infected individual in a completely susceptible population. In the context of our work the basic reproductive number quantifies the number of new believers that are converted, from an entire population of unbelievers, by one single believer during the time in which he/she holds that ideology. Under the assumptions of our model, this number will depend on the trade-off between transmissibility and the average recovery rate of each core group. In other words, it will depend on the trade-off between investment (time, money, etc.) on recruitment and investment on retention [10],[3]. The core group with the higher basic reproductive number will drive the other core group to extinction, for it will seize all the individuals in the general population, $G(t)$ until there are no more individuals left to convert or recruit. Surviving in this case implies keeping a good balance between recruitment and retention. Recall that the efforts that the core groups $C_{1}(t)$ and $C_{2}(t)$ put into recruitment are quantified by $q \beta_{1}$ and $(1-q) \beta_{1}$, respectively, and their cost of retention is measured by the minimum percapita recovery rate of the subclasses embedded in these core groups, $\gamma=\min \left\{\gamma_{1}, \gamma_{2}, \gamma_{3}\right\}$ and $\alpha=\min \left\{\alpha_{1}, \alpha_{2}, \alpha_{3}\right\}$. Thus longer resident times in the subclasses, $S_{i}(t), E_{i}(t)$, and $F_{i}(t)$, for $i=1,2$ increase the cost of retention for the respective fanatic groups.

Theorem 2.2 Let $\widetilde{R}_{3}=\frac{\beta_{3}}{\gamma_{3}+\mu}<1$ and $\widehat{R}_{3}=\frac{\rho_{3}}{\alpha_{3}+\mu}<1$. Then $F_{1}(t) \rightarrow 0, F_{2}(t) \rightarrow 0$ as $t \rightarrow \infty$.

Proof: We consider the $F_{1}$ and $F_{2}$ equations:

$$
\begin{aligned}
\frac{d F_{1}}{d t} & =-\left(\gamma_{3}+\mu\right) F_{1}\left(-\left(\frac{\beta_{3}}{\gamma_{3}+\mu}\right) \frac{E_{1}}{C_{1}}+1\right) \\
& =-\left(\gamma_{3}+\mu\right) F_{1} \frac{E_{1}}{C_{1}}\left(\frac{C_{1}}{E_{1}}-\left(\frac{\beta_{3}}{\gamma_{3}+\mu}\right)\right)=-\left(\gamma_{3}+\mu\right) F_{1} \frac{E_{1}}{C_{1}}\left(\frac{C_{1}}{E_{1}}-\widetilde{R}_{3}\right) \\
\frac{d F_{2}}{d t} & =-\left(\alpha_{3}+\mu\right) F_{2} \frac{E_{2}}{C_{2}}\left(\frac{C_{2}}{E_{2}}-\widehat{R}_{3}\right) .
\end{aligned}
$$

We observe that $\frac{C_{i}}{E_{i}} \geq 1, i=1,2$. The claim follows immediately.

To obtain the conditions under which the fanatic groups $F_{i}(t)$ become established, we analyze the stability of the $D F E$ by considering the dynamics of the subsystem before fanatic groups are able to rise. We let

$$
\widetilde{R}_{i}=\frac{\beta_{i}}{\gamma_{i}+\mu}, i=1,2,3, \quad \widehat{R}_{1}=\frac{\beta_{1}}{\alpha_{1}+\mu}, \quad \widehat{R}_{i}=\frac{\rho_{i}}{\alpha_{i}+\mu}, i=2,3, \quad \text { with } \quad \widetilde{R}_{3}<1, \quad \widehat{R}_{3}<1
$$

The following theorem gives conditions for the stability of the three $D F E$.

Theorem 2.3 With $\widetilde{R}_{i}, \widehat{R}_{i}$ defined as in (18), we have

(i) $D F E_{0}$ is stable when $\widetilde{R}_{1}<\frac{1}{q}$ and $\widehat{R}_{1}<\frac{1}{1-q}$

(ii) DFE $E_{1}$ is stable when $\frac{1}{q}<\widetilde{R}_{1}$ and $\widetilde{R}_{2}<1$

(iii) DFE $E_{2}$ is stable when all three conditions $\widetilde{R}_{1} q<(1-q) \widehat{R}_{1}, \frac{1}{1-q}<\widehat{R}_{1}$, and $\widehat{R}_{2}<1$ are satisfied. 
Proof: We can prove the stability of $D F E_{i}$ by examination of their eigenvalues. Let $\lambda_{i j}$ represent the eigenvalues of the $D F E_{i}$ in the subsystem given by equations (8) -(11). We obtain

$$
\begin{aligned}
\lambda_{01}= & -\left(\gamma_{1}+\mu\right)+\beta_{1} q, \lambda_{02}=-\left(\alpha_{1}+\mu\right)+\beta_{1}(1-q), \lambda_{03}=-\left(\gamma_{2}+\mu\right), \\
& \lambda_{04}=-\left(\alpha_{2}+\mu\right) \\
\lambda_{11}= & -\beta_{1} q+\left(\gamma_{1}+\mu\right), \lambda_{12}=-\left(\alpha_{1}+\mu\right)-\left(\gamma_{1}+\mu\right)+\frac{\gamma_{1}+\mu}{q}, \\
& \lambda_{13}=\beta_{2}-\left(\gamma_{2}+\mu\right), \lambda_{14}=-\left(\alpha_{2}+\mu\right) \\
\lambda_{21}= & \frac{q}{1-q}\left(\alpha_{1}+\mu\right)-\left(\gamma_{1}+\mu\right), \lambda_{22}=-\beta_{1}(1-q)+\left(\alpha_{1}+\mu\right), \\
& \lambda_{23}=-\left(\gamma_{2}+\mu\right), \lambda_{24}=\rho_{2}-\left(\alpha_{2}+\mu\right)
\end{aligned}
$$

The theorem follows by requiring $\lambda_{i j}<0$.

The stability of each $D F E$ together with bifurcation analysis predicts that in the long run at most one of the ideologies will persist. The equilibria $D F E_{i}, i=1,2$ are born in forward bifurcations with $D F E_{0}$. In the subsystem under consideration, there also exist two endemic equilibria, $E E_{1}$ and $E E_{2}$ where an ideology has already infiltrated the population and is to thriving among the group of vulnerable (or naive) individuals, $S_{i}(t)$ as well as among the group of semi-fanatics, $E_{i}(t)$. These endemic equilibria are involved in bifurcations with $D F E_{i}, i=1,2$, respectively, and are given by

$$
\begin{aligned}
E E_{1}: \quad & \left\{S_{1}=\frac{\left(\gamma_{2}+\mu\right)\left(\beta_{1} q \beta_{2}-\gamma_{1} \gamma_{2}-\gamma_{2} \beta_{2}+\gamma_{2}^{2}+\gamma_{2} \mu-\gamma_{1} \mu-\beta_{2} \mu\right)}{\beta_{1} q \beta_{2}^{2}}, S_{2}=0\right\} \\
& \left\{E_{1}=\frac{-\left(\beta_{1} q \beta_{2}-\gamma_{1} \gamma_{2}-\gamma_{2} \beta_{2}+\gamma_{2}^{2}+\gamma_{2} \mu-\gamma_{1} \mu-\beta_{2} \mu\right)\left(-\beta_{2}+\gamma_{2}+\mu\right)}{\beta_{1} q \beta_{2}^{2}}, E_{2}=0\right\} \\
E E_{2}: \quad & \left\{S_{1}=0, S_{2}=\frac{\left(\alpha_{2}+\mu\right)\left(\beta_{1} q \rho_{2}-\beta_{1} \rho_{2}+\alpha_{2} \rho_{2}-\alpha_{2}^{2}+\alpha_{1} \alpha_{2}+\rho_{2} \mu-\alpha_{2} \mu+\alpha_{1} \mu\right)}{\beta_{1} \rho_{2}^{2}(q-1)}\right\}, \\
& \left\{E_{1}=0, E_{2}=\frac{-\left(\beta_{1} q \rho_{2}-\beta_{1} \rho_{2}+\alpha_{2} \rho_{2}-\alpha_{2}^{2}+\alpha_{1} \alpha_{2}+\rho_{2} \mu-\alpha_{2} \mu+\alpha_{1} \mu\right)\left(-\rho_{2}+\alpha_{2}+\mu\right)}{\beta_{1} \rho_{2}^{2}(q-1)}\right\}
\end{aligned}
$$

Theorem 2.4 The equilibria $E E_{1}$ and $D F E_{1}$ undergo a forward transcritical bifurcation when $\widetilde{R}_{2}=1$ with the E $E_{1}$ existing biologically when $\widetilde{R}_{2}>1$. Similarly, the equilibria E $E_{2}$ and DFE $E_{2}$ undergo a forward trancritical bifurcation when $\widehat{R}_{2}=1$ with the E $E_{2}$ existing biologically when $\widehat{R}_{2}>1$

Proof: To prove the statement about $E E_{1}$ and $D F E_{1}$, we first observe that $E E_{1}=D F E_{1}$ when $\gamma_{2}+\mu=\beta_{2}$, i.e., when $\widetilde{R}_{2}=1$. We first rewrite $E E_{1}$ in terms of $\widetilde{R}_{1}$ and $\widetilde{R}_{2}$. The $E_{1}$ coordinate of $E E_{1}$ is

$$
E_{1}=\frac{\gamma_{1}\left(q \widetilde{R}_{1} \widetilde{R}_{2}-1\right)+\gamma_{1}\left(1-\widetilde{R}_{2}\right)+\mu \widetilde{R}_{2}\left(q \widetilde{R}_{1}-1\right)}{\widetilde{R}_{2}^{2} \widetilde{R}_{1}\left(\gamma_{1}+\mu\right)} .
$$

We let $\widetilde{R}_{1}=\frac{1}{q}+\delta$ and $\widetilde{R}_{2}=1+\epsilon$, where $\delta$ is not necessarily small but $|\epsilon| \ll 1$. Expanding in $\epsilon$ gives

$$
E_{1}=\frac{\delta q \epsilon}{1+\delta q}+O\left(\epsilon^{2}\right)
$$


Thus for $\epsilon<0$, we have $\widetilde{R}_{2}<1 \Rightarrow E_{1}<0$ whereas for $\epsilon>0$, we have $\widetilde{R}_{2}>1 \Rightarrow E_{1}>0$. For $E E_{2}$, we similarly rewrite the expression for $E_{2}$ in terms of $\widehat{R}_{1}$ and $\widehat{R}_{2}$ :

$$
E_{2}=\frac{\left[\alpha_{2}^{2}\left(\widehat{R}_{2}-1\right)+\left(\mu^{2}+\mu \alpha_{1}+\mu \alpha_{2} \widehat{R}_{2}\right)\left[1-(1-q) \widehat{R}_{1}\right]+\alpha_{1} \alpha_{2}\left[1-(1-q) \widehat{R}_{1} \widehat{R}_{2}\right]\right] \alpha_{2}\left(\widehat{R}_{2}-1\right)}{\widehat{R}_{1}\left(\alpha_{1}+\mu\right)\left(\widehat{R}_{2} \alpha_{2}+\mu\right)^{2}(q-1)} .
$$

We let $\widehat{R}_{1}=\frac{1}{1-q}+\delta$ and $\widehat{R}_{2}=1+\epsilon$, where $\delta$ is not necessarily small but $|\epsilon| \ll 1$. Expanding in $\epsilon$ gives

$$
E_{2}=\frac{\delta(1-q) \alpha_{2} \epsilon}{1+\delta(1-q)\left(\mu+\alpha_{2}\right)}+O\left(\epsilon^{2}\right)
$$

Thus for $\epsilon<0$, we have $\widehat{R}_{2}<1 \Rightarrow E_{2}<0$ whereas for $\epsilon>0$, we have $\widehat{R}_{2}>1 \Rightarrow E_{2}>0$. The theorem follows since the structure of $S_{1}$ and $S_{2}$ in the respective equilibria differs only by a factor related to $\widetilde{R}_{2}$ and $\widehat{R}_{2}$, respectively.

This is again consistent with the persistence of only one ideology. The core group that maximizes their basic reproductive number will prevail. Building a critical mass of individuals committed to the ideology and sustaining it is crucial for the existence and survivability of the organization [3],[12]. The results of this mathematical model reveal that without cross interaction of population being recruited by two organizations (in other words the ability to take over from the other core group or share prospective members) both fanatic ideologies cannot exist in the long run. In the next section we show that in order for persistence of both fanatic ideologies, we need to have cross interaction of the populations being recruited by the two ideologies. Including additional trade-offs between recovery rate and competitive ability (ability to take over individuals/resources from the other core groups or share resources, i.e., the presence of cross interaction) may not necessarily favor the fanatic group with the largest reproductive number and may in fact support coexistence of multiple fanatic groups and organizations [1].

\section{Description of the cross interaction model}

We have so far considered an ideal situation in which the environmental conditions are not changing, the population is homogeneous (i.e., the species are identical with identical limiting factors ecological preferences), and there is no interaction between the two core groups. We now consider modifications to the model where our new equations describe cross interaction of the competing ideologies. Cross interaction (cross recruitment), recovery and demography are mechanisms responsible for the competitive exclusion and/ or coexistence of two related social groups (species)[7]. In an attempt to better understand the key components of that lead to the survival of social organizations we now extend the model to consider cross interaction of competing ideologies. The governing 
equation of this new model are given by:

$$
\begin{aligned}
\frac{d G}{d t}= & \Lambda+\gamma_{3} F_{1}+\gamma_{2} E_{1}+\alpha_{2} E_{2}+\alpha_{3} F_{2}+\gamma_{1} S-\frac{\beta_{1} G C}{N}-\mu G \\
\frac{d S}{d t}= & \frac{\beta_{1} G C}{N}-\frac{\beta_{2} S\left(E_{1}+q E_{2}+F_{1}\right)}{C}-\frac{\rho_{2} S\left(q E_{1}+E_{2}+F_{2}\right)}{C}-\gamma_{1} S-\mu S \\
\frac{d E_{1}}{d t}= & \frac{\beta_{2} S\left(E_{1}+q E_{2}+F_{1}\right)}{C}-\gamma_{2} E_{1}-\frac{\beta_{3} E_{1} F_{1}}{C}+\frac{\rho_{4} E_{2}\left(q E_{1}+F_{1}\right)}{C}-\frac{\beta_{4} E_{1}\left(q E_{2}+F_{2}\right)}{C} \\
& -\mu E_{1}-\frac{\beta_{5} E_{1} F_{2}}{C} \\
\frac{d E_{2}}{d t}= & \frac{\rho_{2} S\left(q E_{1}+E_{2}+F_{2}\right)}{C}-\alpha_{2} E_{2}-\frac{\rho_{3} E_{2} F_{2}}{C}-\frac{\rho_{4} E_{2}\left(q E_{1}+F_{1}\right)}{C}+\frac{\beta_{4} E_{1}\left(q E_{2}+F_{2}\right)}{C} \\
& -\mu E_{2}-\frac{\rho_{5} E_{2} F_{1}}{C} \\
\frac{d F_{1}}{d t}= & \frac{\beta_{3} E_{1} F_{1}}{C}-\gamma_{3} F_{1}-\mu F_{1}+\frac{\rho_{5} E_{2} F_{1}}{C} \\
\frac{d F_{2}}{d t}= & \frac{\rho_{3} E_{2} F_{2}}{C}-\alpha_{3} F_{2}-\mu F_{2}+\frac{\beta_{5} E_{1} F_{2}}{C}
\end{aligned}
$$

where all parameters are nonnegative and where the total population, $N(t)$, and the core group, $C(t)$, are define as

$$
\begin{aligned}
& N=G+S+E_{1}+E_{2}+F_{1}+F_{2} \\
& C=S+E_{1}+E_{2}+F_{1}+F_{2} .
\end{aligned}
$$

For simplicity we set the parameters $\beta_{5}$ and $\rho_{5}$ to zero. We see that one of the main differences between this and the previous model is that the vulnerable individuals are initially under the influence of both ideologies. At this first stage of conversion the two ideologies seem to be very similar and indistinguishable from the perspective of the vulnerable, naive, and susceptible individuals in the $S(t)$ group. Both ideologies initiate the conversion of naive individuals but as the individuals starts to become more committed the ideologies diverge to distinct ideas and only one of the ideologies can now be influential. However, these two distinct ideologies still share some parallel characteristic and thus they are able to recruit members from the semi-fanatic group of the other organization and convert them. A pictorial illustration of this model is given in Figure 2.

Similar to the previous model we can mathematically formulate the existence of at least one fanatic group for some given parameter by considering the core population. The establishment of the core population can be stated in the following theorem:

Theorem 3.1 Let

$$
\gamma=\min \left\{\gamma_{1}, \gamma_{2}, \gamma_{3}, \alpha_{2}, \alpha_{3}\right\}
$$

and define

$$
R_{1}=\frac{\beta_{1}}{\gamma+\mu}
$$

Suppose $R_{1}<1$. Then $C \rightarrow 0$ as $t \rightarrow \infty$. 


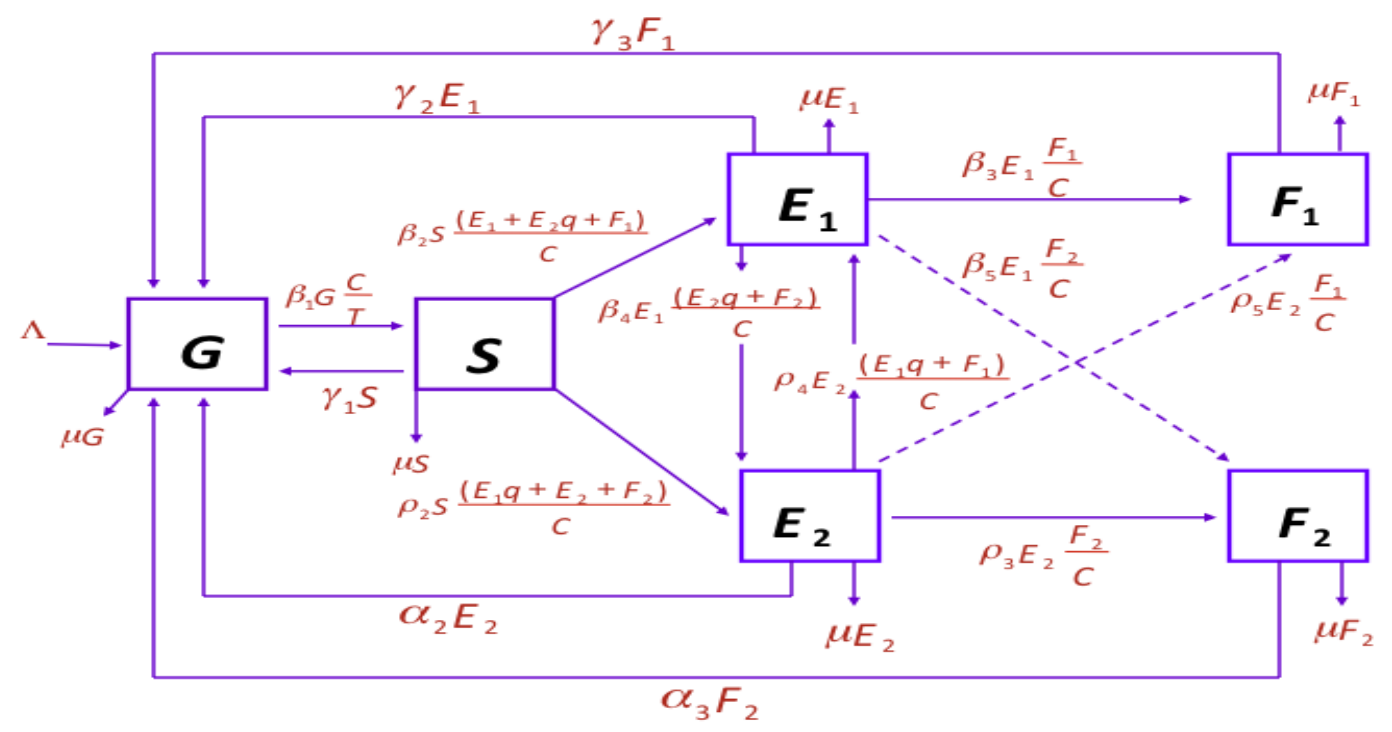

Figure 2: Flow diagram for the cross interaction model.

Proof: Adding the relevant equations gives

$$
\begin{aligned}
\frac{d C}{d t} & \leq-C\left(\beta_{1} C+\gamma+\mu-\beta_{1}\right) \\
& =\beta_{1}(1-C) C-(\gamma+\mu) C \\
& =C\left(\beta_{1}(1-C)-(\gamma+\mu)\right) \\
& =C\left((1-c) \frac{\beta_{1}}{\gamma+\mu}\right)(\gamma+\mu) \\
& =C(\gamma+\mu)\left(\frac{\beta_{1}}{\gamma+\mu}(1-C)-1\right) \\
& <(\gamma+\mu) \frac{\beta_{1}}{\gamma+\mu} C-(\gamma+\mu) C \\
& =C(\gamma+\mu)\left(R_{1}-1\right)
\end{aligned}
$$

The conclusions follow from the assumptions of the theorem. $\square$

As before, we can examine the disease-free equilibria for the cross recruiting model:

$$
\begin{array}{ll}
D F E_{1}: & \left\{G=\frac{\Lambda}{\mu}, S=0\right\} \\
D F E_{2}: & \left\{G=\frac{\Lambda\left(\gamma_{1}+\mu\right)}{\beta_{1} \mu}, S=\frac{-\Lambda\left(-\beta_{1}+\gamma_{1}+\mu\right)}{\beta_{1} \mu}\right\}
\end{array}
$$

Note that setting $\beta_{5}=\rho_{5}=0$ does not change the dynamics of the disease-free equilibria. The previous theorem shows that $D F E_{1}$ will be stable when $R_{1}<1$, since the equilibrium point $D F E_{1}$ 
coincides with the core group's population being zero. If we let $\widetilde{R}_{1}=\frac{\beta_{1}}{\gamma_{1}+\mu}$, then $D F E_{2}$ is given by

$$
\left\{G=\frac{\Lambda}{\widetilde{R}_{1} \mu}, S=\frac{\Lambda}{\mu}\left(1-\frac{1}{\widetilde{R}_{1}}\right)\right\} .
$$

This equilibrium point is relevant to our application only when $\widetilde{R}_{1}>1$ otherwise we will have negative population size. In order to prove its stability, we first consider the case when the fanatic populations, $F_{1}$ and $F_{2}$ go to zero. From equation (26) it follows that if $\frac{\beta_{3}}{\gamma_{3}+\mu}<1$ then $F_{1}(t) \rightarrow 0$, as $t \rightarrow \infty$. Similarly, we see from equation (27) that if $\frac{\rho_{3}}{\alpha_{3}+\mu}<1$ then $F_{2}(t) \rightarrow 0$ as $t \rightarrow \infty$. This can readily be observed by setting $\beta_{5}=\rho_{5}=0$ and rearranging the terms in equations (26) and (27). In considering the stability of $D F E_{2}$, we define

$$
R_{2}=\frac{\beta_{2}}{\gamma_{2}+\mu}, \widetilde{R}_{2}=\frac{\rho_{2}}{\alpha_{2}+\mu}, R_{3}=\frac{\beta_{3}}{\gamma_{3}+\mu}, \widetilde{R}_{3}=\frac{\rho_{3}}{\alpha_{3}+\mu}
$$

and

$$
\begin{aligned}
R_{d} & =\frac{\frac{q^{2} \beta_{2} \rho_{2}}{\left(\gamma_{2}+\mu\right)\left(\alpha_{2}+\mu\right)}+\frac{\beta_{2}}{\gamma_{2}+\mu}+\frac{\rho_{2}}{\alpha_{2}+\mu}}{\frac{\beta_{2} \rho_{2}}{\left(\gamma_{2}+\mu\right)\left(\alpha_{2}+\mu\right)}+1} \\
& =\frac{q^{2} R_{2} \widetilde{R}_{2}+R_{2}+\widetilde{R}_{2}}{R_{2} \widetilde{R}_{2}+1}
\end{aligned}
$$

Theorem 3.2 Let $R_{1}>1, R_{3}<1, \widetilde{R}_{3}<1, R_{2}<1, \widetilde{R}_{2}<1, R_{d}<1$. Then DFE $E_{2}$ is stable.

Proof: Two of the eigenvalues are on the diagonal and are given as

$$
-\left(\gamma_{3}+\mu\right),-\left(\alpha_{3}+\mu\right) .
$$

The remaining submatrix is in block diagonal form with each submatrix being a 2-by-2 matrix. One of these submatrices yields the eigenvalues

$$
-\mu,-\left(\beta_{1}+\gamma_{1}+\mu\right)
$$

The remaining two eigenvalues are determined by the 2-by-2 submatrix

$$
J_{1}=\left(\begin{array}{cc}
\beta_{2}-\gamma_{2}-\mu & \beta_{2} q \\
\rho_{2} q & \rho_{2}-\alpha_{2}-\mu
\end{array}\right)
$$

We will have stability when $\operatorname{tr}\left(J_{1}\right)<0$ and $\operatorname{det}\left(J_{1}\right)>0$. The trace condition is equivalent to $\beta_{2}+\rho_{2}<2 \mu+\gamma_{2}+\alpha_{2}$. The determinant condition is equivalent to $R_{d}<1$.

Claim: $\operatorname{det}\left(J_{1}\right)>0 \Rightarrow \operatorname{tr}\left(J_{1}\right)<0$

Proof of Claim: The condition $R_{d}<1$, i.e., $\operatorname{det}\left(J_{1}\right)>0$ can be rewritten as

$$
\begin{aligned}
\beta_{2}+\rho_{2} & <\frac{1}{\mu}\left(\mu^{2}+\beta_{2} \rho_{2}+\gamma_{2} \alpha_{2}+\gamma_{2} \mu+\mu \alpha_{2}-\beta_{2} q^{2} \rho_{2}-\beta_{2} \alpha_{2}-\gamma_{2} \rho_{2}\right) \\
& \leq \frac{1}{\mu}\left[\mu^{2}+\mu\left(\gamma_{2}+\alpha_{2}\right)+\left(\beta_{2}-\gamma_{2}\right)\left(\rho_{2}-\alpha_{2}\right)\right] \\
& =\mu+\left(\gamma_{2}+\alpha_{2}\right)+\frac{1}{\mu}\left(\beta_{2}-\gamma_{2}\right)\left(\rho_{2}-\alpha_{2}\right) .
\end{aligned}
$$


But $R_{2}<1 \Rightarrow \beta_{2}-\gamma_{2}<\mu$. Similarly $\widetilde{R}_{2}<1 \Rightarrow \rho_{2}-\alpha_{2}<\mu$. Thus the proof now becomes

$$
\begin{aligned}
\beta_{2}+\rho_{2} & <\mu+\left(\gamma_{2}+\alpha_{2}\right)+\frac{1}{\mu}(\mu \mu) \\
& =2 \mu+\gamma_{2}+\alpha_{2} .
\end{aligned}
$$

This is exactly the condition for $\operatorname{tr}\left(J_{1}\right)<0$ and the Claim is proved.

Thus stability of the equilibria will hold when $\operatorname{det}\left(J_{1}\right)>0$ which is exactly the same as requiring $R_{d}<1$.

Corollary 3.1 Let $R_{1}>1, R_{3}<1, \widetilde{R}_{3}<1, R_{2}<1, \widetilde{R}_{2}<1$, and $R_{d}>1$. Then DF $E_{2}$ is unstable.

Proof: Under these conditions, we have $\operatorname{det}\left(J_{1}\right)<0$ which shows the instability.

We now give an interpretation of $R_{d}$, used in the previous theorem and corollary.

The inverse of the denominator of $R_{2}$ above, $\left(\gamma_{2}+\mu\right)$, is the mean time and individual spent in the $E_{1}$ group. The numerator is the rate at which individuals the $S$ group move to $E_{1}$ group due to interactions with individuals in $E_{1}, E_{2}$, and $F_{1}$ groups. Thus, $R_{2}$ is the product of the mean time spent in $E_{1}$ times the rate that $S$ individuals move into $E_{1}$. It quantifies how successful the organization with ideology 1 is in converting vulnerable and naive individuals into new semifanatics, $E_{1}$, as well as retaining them in this group. This success is due to efforts and interactions of members in $E_{1}, E_{2}$, and $F_{1}$ with individuals in $S$ that ultimately adopt ideology 1 . The parallel characteristics of the two ideologies (as limited and few as they might be) from the perspective of the vulnerable and naive individuals in $S$ are such that the two ideologies are very similar and thus followers of ideology 2 , who are not fully committed believers but have adopted the ideology, can convert $S$ individuals to $E_{1}$. Similarly, $\widetilde{R}_{2}$ is the product of the mean time spent in the semi-fanatic group, $E_{2}$, times the rate that $S$ individuals move into the $E_{2}$ class due to interactions with the individuals in the groups, $E_{1}, E_{2}$, and $F_{2}$. It quantifies the success of the organization with ideology 2 in recruiting and retaining individuals in the semi fanatic group $E_{2}$. The expression $q R_{2}$ is the product of the mean time spent in $E_{1}$ times the rate that $S$ individuals move into $E_{1}$ class due to interactions with the member in the semi-fanatic group $E_{2}$. It is a measurement of the success in retention and recruitment into the group $E_{1}$ brought about by interactions with members of group $E_{2}$. Even though individuals in groups $E_{1}$ and $E_{2}$ are committed to some degree to their respective ideologies 1 and 2, they still have not fully embraced or don't fully understand the organization's ideology and thus can be persuaded by fanatics and semi-fanatics of the opposing organization to adopt the other ideology and move to the respective semi-fanatic group. It serves as a way of quantifying the benefit that the interactions of the individuals in $E_{1}$ with those in $S$ bring to the recruitment of $E_{2}$ and the retention efforts as it pertains to this class from the entire organization.

Similarly the expression $q \widetilde{R}_{2}$ is the product of the average residence time in $E_{2}$ times the rate that $S$ individuals move into $E_{2}$ due to interactions with members of $E_{1}$. In the numerator of $R_{d}$ we see the effectiveness of the ideologies in thriving among the semi-fanatics. It illustrates the success of converting members of $S$ to believers of the two ideologies and maintaining their commitment. This depends on the independent success of both organizations (represented by the $R_{2}$ and $\widetilde{R}_{2}$ terms) as well as the indirect benefit obtained from the semi-fanatics in the opposing organization (represented by $q R_{2}$ and $q \widetilde{R}_{2}$ ). This makes sense because the numerator should depend on how successful the recruiting of $S$ individuals into the $E_{1}$ and $E_{2}$ classes is and the average residence time in both classes, $E_{1}$ and $E_{2}$. The denominator is the product of $R_{2}$ and $\widetilde{R}_{2}$ summed with 1 . The presence of the " +1 " in the denominator indicates that even if there is no cross-recruiting by 

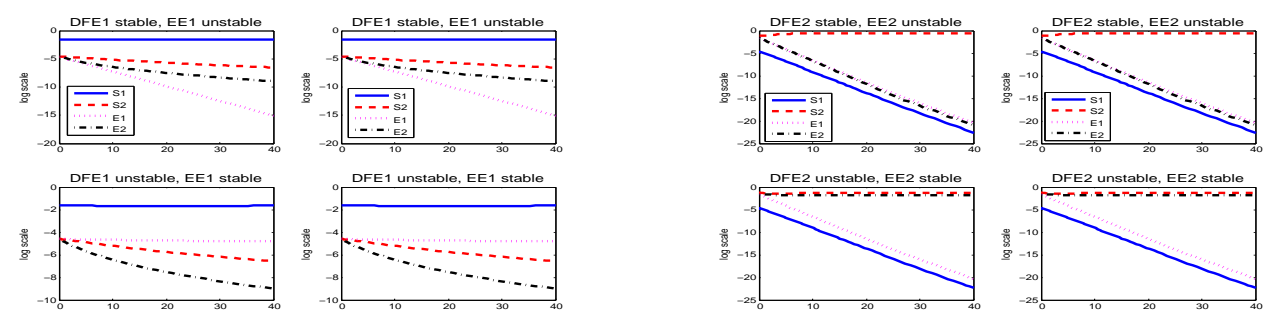

Figure 3: In the top panel of left figure, the DFE1 is stable and both ideologies die off with $\beta_{2}=$ $.25, q=.52, \rho_{2}=.5$ (so that $\widetilde{R}_{2}=\frac{25}{51}$ and $\widetilde{R}_{1}=\frac{200}{81}>\frac{1}{q}$ ). In the bottom panel of the left figure, EE1 is now stable and the first ideology persists with $\beta_{2}=.528, q=.527, \rho_{2}=.5$ (so that $\widetilde{R}_{2} \approx 1.035$ ). The right two plots of the left figure are simply magnifications of the left plots. In the top panel of right figure, the DFE2 is stable and both ideologies die off with $\beta_{2}=.25, q=.01, \rho_{2}=.01$ (so that $\widehat{R}_{2} \approx .0192, \widehat{R}_{1}=\frac{200}{81}>\frac{1}{1-q}$, and $\widetilde{R}_{1}=\frac{200}{81}$ ). In the bottom panel of the right figure, EE2 is now stable and the second ideology persists with $\beta_{2}=.25, q=.01, \rho_{2}=.85$ (so that $\widehat{R}_{2} \approx 1.635$ ). The right two plots of the right figure are simply magnifications of the left plots. All plots have a vertical log scale in order to see the curves that exponentially go to zero.

the classes with different ideology (i.e., $\beta=0$ or $\rho=0$ ), it's still possible to have $R_{d}>1$. This again makes intuitive sense. In such a case the total combine success of both ideologies depends on the sum of their relative success, quantify by $R_{2}$ and $\widetilde{R}_{2}$. The cross-recruiting increases $R_{d}$ (since $q \neq 0$ ), whereas the lack of cross-recruiting (i.e., $q=0$ ) changes $R_{d}$ in a natural way.

\section{Numerical Results}

The key difference between the two models is whether individuals are allowed to switch between ideologies. Figure 3 demonstrates the results of Theorem 2.4 in which only one of the ideologies is able to persist. In each case, there is a critical threshold in which a single ideology can take hold. In both subfigures, we have

$$
\begin{array}{r}
\gamma_{1}=\frac{1}{50}, \gamma_{2}=\frac{13}{50}, \gamma_{3}=\frac{1}{4}, \quad \beta_{1}=\frac{2}{3}, \beta_{3}=\frac{1}{2} \\
\mu=\frac{1}{4}, \rho_{1}=\frac{1}{2}, \rho_{3}=\frac{1}{2}, \alpha_{1}=\frac{1}{50}, \alpha_{2}=\frac{27}{100}, \alpha_{3}=\frac{1}{4},
\end{array}
$$

and we vary $\beta_{2}, \rho_{2}, q$ as described in the captions. The left plot of Figure 3 demonstrates the conditions under which the first ideology is established whereas the right plot of Figure 3 shows the conditions under which the second ideology is established.

In contrast to the results of the non-cross interaction model, the cross interaction model permits the co-existence of competing ideologies. This is a key difference between the two models as this coexistence is observed in many real world systems with competing ideologies. Figure 4 demonstrates 

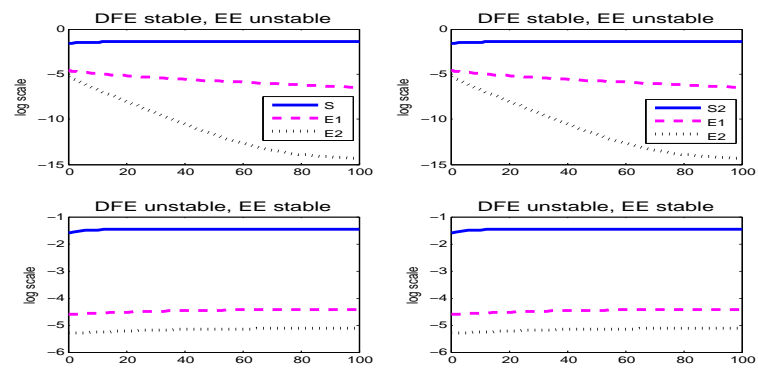

Figure 4: In the top panel, the $D F E$ is stable and both ideologies die off with $q=.0001$ (so that $\left.R_{d} \approx .99961\right)$. In the bottom panel, the $\mathrm{EE}$ is now stable and the both ideologies persist with $q=.2$ (so that $R_{d} \approx 1.0146$ ). The right two plots of the figure are simply magnifications of the left plots. All plots have a vertical log scale in order to see the curves that exponentially go to zero.

the results of Theorem 2.6 and its Corollary. With parameter values

$$
\begin{array}{r}
\gamma_{1}=\frac{1}{4}, \gamma_{2}=\frac{13}{50}, \gamma_{3}=\frac{1}{4}, \beta_{1}=\frac{2}{3}, \beta_{2}=\frac{1}{2}, \beta_{3}=\frac{2}{5}, \beta_{4}=\frac{1}{2}, \beta_{5}=\frac{1}{2}, \\
\mu=\frac{1}{4}, \rho_{2}=\frac{4}{10}, \rho_{3}=\frac{2}{5}, \rho_{4}=\frac{51}{100}, \rho_{5}=\frac{1}{2}, \alpha_{2}=\frac{27}{100}, \alpha_{3}=\frac{1}{4},
\end{array}
$$

we can vary the parameter $q$ to go from a state in which the DFE is the only stable state to one in which an endemic equilibrium, and hence both ideologies, exists.

\section{Conclusions}

This paper considers two models in which the population is divided into non-core and core groups. The first model considered the case of two core populations with no cross interaction between the subpopulations and both core groups draw from the same general population. In contrast, the second model incorporates cross interactions between the two core groups with each core group trying to draw from the same susceptible population and from the other core group. The two approaches model the role of competing ideologies within the subpopulations as each tries to address how an ideology is able to establish itself when it infiltrates a core group or is not able to establish itself if either the reruitment is too slow or the retention is not adequate. Both models also allow for a recovery from the fanatic ideology with a return to the general population. In both models, we were able to prove stability conditions about the various disease free equilibria and, in particular, that establishment of the core group of an ideology is necessary for persistence of that ideology.

In the case of the non-cross interaction model, we saw competition between the core groups for members and we saw that success depended on the trade-off between investment on recruitment 
and investment on retention. The core group with the higher basic reproductive number will drive the other core group to extinction, which is consistent with the persistence of only one ideology. Building a critical mass of individuals committed to the ideology and sustaining it is crucial for its existence and survivability. The results of this mathematical model reveal that without cross interaction of populations being recruited by two organizations (in other words the ability to take over from the other core group or share prospective members) both fanatic ideologies cannot co-exist in the long run.

For the cross interaction model, we showed that in order to have persistence of both fanatic ideologies, we need to have cross interaction of the populations being recruited by the two ideologies. This gave one of the main results of this study: that cross interactions are required in order to achieve coexistence of competing fanatic ideologies. We showed including additional trade-offs between recovery rate and competitive ability, that is, the ability to take over individuals from the other core groups, may not necessarily favor the fanatic group with the largest reproductive number and may in fact support coexistence of multiple fanatic groups and organizations.

For both models, the more efforts that an organization implements to recruit and retain its members the more effective it will be in transmitting the ideology to other individuals but this holds for homogeneous populations. Our model focuses on long-term dynamics and it is possible that transient dynamics might be significant in changing environments. Future work on this topic could incorporate more realism within the models such as including volatile or variable environmental conditions. Additionally, in the cross-diffusion model, we could allow for cross-recruitment from the $E_{i}$ to $F_{j}\left(\beta_{5}, \rho_{5} \neq 0\right)$. We could also allow for backsliding where individuals move back one class when in the $E$ category only (rather than exit to the general population). We could also imagine explicitly incorporating intervention or re-education programs. For example, a recent article describes a jihadi rehabilitation center that attempts to do just that [27]. Allowing for additional competing ideologies, ideologies with distinct processes, limited resources of a given ideology, or heterogeneous populations (where individual actions would play a role) would also be natural extensions.

\section{Acknowledgments}

A special thanks goes to Carlos Castillo-Chavez for many helpful discussions in the creation of this model and Norman Johnson for helpful discussion on the interpretations. The author also wishes to thank the anonymous reviewer for constructive insights into the model. 


\section{References}

[1] Adler, F. R., Mosquera, J. Super- and Coinfection: Filling the Range, In: Adaptive Dynamics of Infectious Diseases: In Pursuit of Virulence Management, U. Dieckmann, JAJ. Metz, MW Sabelis, and K. Sigmund (eds.), pp. 138-149, Cambridge University Press, 2002.

[2] Ausloos, M. and Petroni, F., Statistical dynamics of religion evolutions, Physica A, 388: 44384444, 2009.

[3] Bremermann, H. J. and Thieme, H. R., A competitive exclusion principle for pathogen virulence, Journal of Mathematical Biology, 27:179-190, 1989.

[4] Bowers, R. G., Community dynamics, invasion criteria and the co-evolution of host pathogen, Complexity International, vol.08, 2000.

[5] Boyd, R. and Richerson, P., Culture and the evolutionary process, The University of Chicago Press, Chicago, 1976.

[6] Caram, L. F., Caiafa, C. F., Proto,A. N., and Ausloos, M., Dynamic peer-to-peer competition., Physica A, 389:2628-2636, 2010.

[7] Castillo-Chavez, C. and Velasco-Hernandez, J. X., On the Relationship Between Evolution of Virulence and Host Demography, Journal of theoretical Biology, 192:437-444, 1998.

[8] Castillo-Chavez, C., and Song, B., Models for the Transmission Dynamics of Fanatic Behaviors, in: Bioterrorism: Mathematical Modeling Applications in Homeland Security (SIAM Frontier Series in Applied Mathematics), pp.155-172, H. T. Banks and C. Castillo-Chavez (eds.), SIAM, Philadelphia, PA, 2003.

[9] Dennett, D., Darwin's dangerous idea, Simon \& Schuster, New York, London, Toronto, Sydney, Tokyo, Singapore, 1995.

[10] Ganusov, V. V. and Rustom, A., Trade-offs and the evolution of virulence of microparasites: do details matter?, Theoretical Population Biology, 64: 211-220, 2003.

[11] Hadeler, K. P. and Castillo-Chavez, C., A Core Group Model for Disease Transmission. J. Math. Biosci., 128:41-55, 1995.

[12] Hayward, J. A General Model of Church Growth and Decline Journal of Mathematical Sociology, 29:177-207, 2005.

[13] Hethcote, H. W. and Yorke, J. A., Gonorrehea transmission dynamics and control, Lecture Notes in Biomath. 56:1-105, Springer-Verlag, Berlin, 1984.

[14] Hsu, B. S., Cheng, K. S., and Hubbell, P., Exploitative Competitive of Microorganism for Two Complementary Nutrients in Continuous Cultures, SIAM Journal on Applied Mathematics, vol.41, 3:422-444, 1981.

[15] Levin, S. A., Community Equilibria and Stability, and an Extension of the Competitive Exclusion Principle, America Naturalist, vol.104, 39:413-423, 1970. 
[16] Loladze, I., Kuang, Y., Elser, J. J., Fagan, W. F., Coexistence of two predators on one prey mediated by stoichimetry, Theoretical Population Biology, 65:1-15, 2003.

[17] May, R. M.,Stability and Complexity in Model Ecosystems, Princeton University Press, 1973.

[18] Newhard, J.M., The Genesis of the State: Mathematical Models of Conflict and Cooperation, Masters Thesis for Bowling Green State University, May 2009.

[19] Queiroz, J. and El-Hani, C.N., Towards a Multi-level Approach to the Emergence of Meaning Processes in Living Systems, Acta Biotheoretica, 54: 179-206, 2006.

[20] Salthe, S. N., Evolving Hierarchical Systems: Their Structure and Representation, Columbia University Press, 1985.

[21] Salthe, S.N., Notes toward a formal history of the levels concept, In. G. Greenberg and E. Tobach (eds.) Evolution of Social Behavior and Integrative Levels, Erlbaum, 1988.

[22] Salthe, S.N., Two forms of hierarchy theory in Western discourses, International Journal of General Systems, 18: 251-264, 1991.

[23] Salthe, S.N., Development and Evolution: Complexity and Change in Biology, MIT Press, 1993.

[24] Santonja, F.J., Tarazona, A.C., and Villanueva, R.J., A mathematical model of the pressure of an extreme ideology on a society, Computers and Mathematics with applications, 56: 836-846, 2008 .

[25] Song, B., Dynamical epidemical models and their applications, Ph.D. Dissertation, Cornell University, Ithaca NY, 2003

[26] Stauffer, D. and Sahimi, M., Discrete simulation of the dynamics of spread of extreme opinions in a society, Physica A, 364: 537-543, 2006.

[27] Temple-Raston, D. Pakistan's Ambitious Program To Re-Educate Militants, April 1, 2013, National Public Radio, Accessed April 1, 2013 from http://www.npr.org/2013/04/01/ 175706661/pakistans-ambitious-program-to-re-educate-militants.

[28] Wang, R. Competition in a patchy environment with cross-diffusion in a three-dimensional Lotka-Volterra system, Nonlinear Analysis: Real World Applications,11:2726-2731, 2010.

[29] Xie, J., Sreenivasan, S., Korniss, G., Zhang, W., Lim, C., and Szymansk, B. K., Social consensus through the influence of committed minorities, Physical Review E, 84: 011130, 2011. 